University of Nebraska - Lincoln

DigitalCommons@University of Nebraska - Lincoln

1998

\title{
Life-history decisions under predation risk: Importance of a game perspective
}

Amos Bouskila

Simon Fraser University, bouskila@bgu.ac.il

Michael E. Robinson

Simon Fraser University

Bernard D. Roitberg

Simon Fraser University, roitberg@sfu.ca

Brigitte Tenhumberg

University of Nebraska - Lincoln, btenhumberg2@unl.edu

Follow this and additional works at: https://digitalcommons.unl.edu/bioscifacpub

Part of the Life Sciences Commons

Bouskila, Amos; Robinson, Michael E.; Roitberg, Bernard D.; and Tenhumberg, Brigitte, "Life-history decisions under predation risk: Importance of a game perspective" (1998). Faculty Publications in the Biological Sciences. 211.

https://digitalcommons.unl.edu/bioscifacpub/211

This Article is brought to you for free and open access by the Papers in the Biological Sciences at DigitalCommons@University of Nebraska - Lincoln. It has been accepted for inclusion in Faculty Publications in the Biological Sciences by an authorized administrator of DigitalCommons@University of Nebraska - Lincoln. 


\title{
Life-history decisions under predation risk: Importance of a game perspective
}

\author{
Amos Bouskila,* Michael E. Robinson, \\ Bernard D. Roitberg, and Brigitte Tenhumberg ${ }^{\dagger}$ \\ Department of Biological Sciences, Simon Fraser University, \\ 8888 University Drive, Burnaby, British Columbia V5A 1S6, Canada \\ Corresponding author - B. D. Roitberg, email roitberg@sfu.ca \\ * Present address: Department of Life Sciences, Ben Gurion University of the Negev, 84105 Beer Sheva, Israel. \\ $\dagger$ Present address: School of Biological Sciences, University of Nebraska-Lincoln, Lincoln, NE 68588, USA
}

\begin{abstract}
We model ontogenetic shifts (e.g. in food or habitat use) during development under predation risk. We ask whether inclusion of state and frequency dependence will provide new insights when compared with game-free life-history theory. We model a simple biological scenario in which a prey animal must switch from a low-predation, low-growth habitat to a high-predation, high-growth habitat. To assess the importance of frequency dependence, we compare the results of four scenarios of increasing complexity: (1) no predation; (2) constant predation; (3) frequency-dependent predation (predation risk diluted at high prey density); and (4) frequency-dependent predation as in (3) but with predators allowed to respond adaptively to prey behavior. State dependence is included in all scenarios through initial size, assumed to be environmental. A genetic algorithm is used to search for optimal solutions to the scenarios. We find substantially different results in the four different scenarios and suggest a decision tree by which biological systems could be tested to ascertain which scenario is most applicable.
\end{abstract}

Keywords: development, frequency dependence, genetic algorithm, ontogenetic shifts, predation risk

\section{Introduction}

Many animals exhibit ontogenetic shifts in food and habitat use as they increase in size (Rowe and Ludwig, 1991), and this is not limited to animals that undergo metamorphosis. Due to these shifts, reptiles, amphibians, fish and invertebrates are often exposed to different growth rates and different predation pressures during different stages of their development (reviewed in Werner and Gilliam, 1984). Models predicting the time and body size for switching between stages have traditionally dealt with developmental constraints (e.g. Smith-Gill and Berven, 1979) or with growth rates and mortality due to environmental conditions (Wilbur and Collins, 1973). Werner and Gilliam (1984) and Abrams and Rowe (1996) introduced the trade-off between growth and predation risk as a predictor for the ontogenetic shift, and Ludwig and Rowe (1990) demonstrated the importance of state and time constraints on such shifts by individuals. Incorporation of the latter two factors helps explain within-population variation in ontogenetic shifts.

The significance of the above advances in evolutionary theory notwithstanding, all of these theories assume that predation parameters vary independently from prey behavior. This is a cause for concern given that recent developments in game theory have shown that the evolutionary pay-off for responses by individual predators or prey may vary as a function of the frequency and form of responses by other 


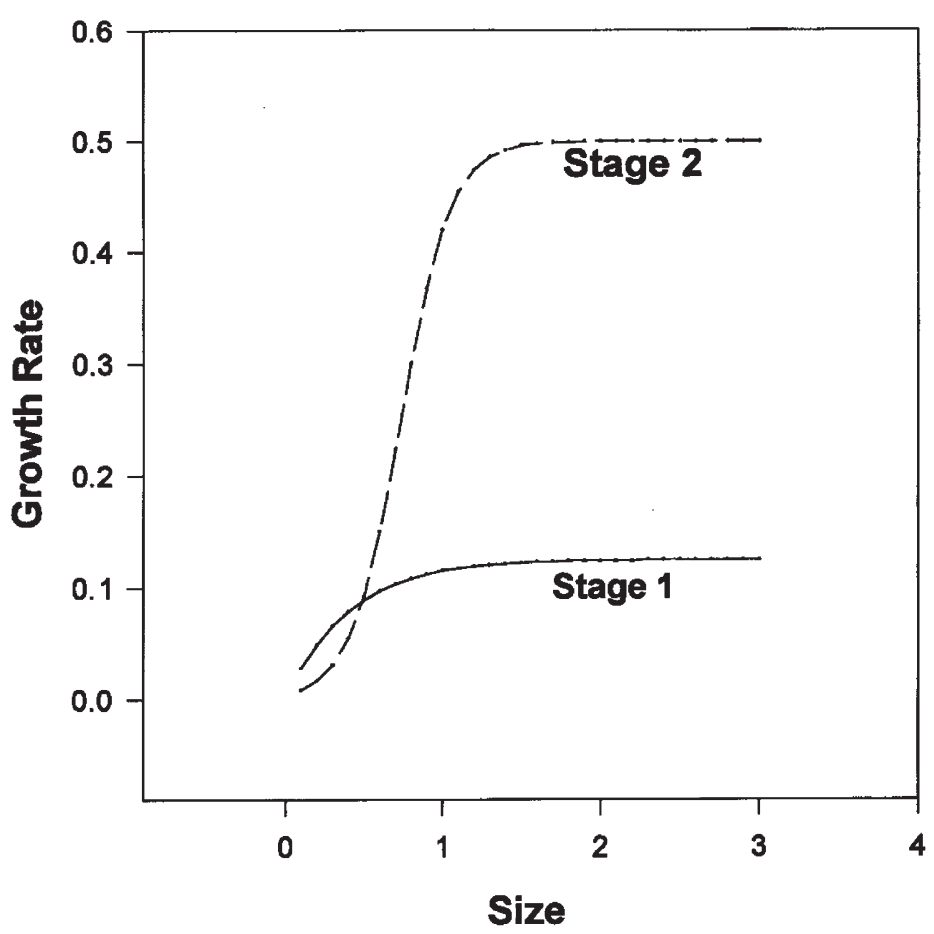

Figure 1. Growth rate as a function of size for stage 1 and stage 2. The point at which the two curves intersect is the point at which a growth-maximizing animal will switch from stage 1 to stage 2 .

members (potentially both predators and prey) of the association. Furthermore, empirical evidence supports the notion that prey and their predators are much more plastic than early theory assumed (Arnold and Wassersug, 1978; Werner et al., 1983; Murdoch, 1994). Thus, if individual activities or developmental decisions at a particular time or place are dependent upon activity levels of predators and other prey (Bouskila, 1995), this might cause us to re-evaluate current theories on ontogenetic shifts. For example, individuals might shift habitats earlier than they would otherwise if they were to receive significant benefits from dilution effects of synchronized habitat shifts (equivalent to the advantages of group formation; see Turner and Pitcher, 1986; Wrona and Dixon, 1991). On the other hand, concomitant responses by predators to such shifts could negate associated benefits (Turner and Pitcher, 1986).

The notion of incorporating both game and state aspects into theories on ontogeny is appealing in that they greatly increase the realism of such theories (Iwasa and Levin, 1995). What is not clear, however, is whether incorporation of these aspects will provide new insights into such theories and, furthermore, whether incorporation of frequency and state dependence is necessary for studying most or only a small subset of developmental phenomena. Thus, our goal in this paper is to study developmental decisions from a variety of frequency-dependent and -independent perspectives and to evaluate their usefulness as components in ontogeny theory.

\section{The biological scenario}

Consider a prey (or host) which sequentially resides in a seasonal environment in two distinct juvenile stages and faces a time horizon regarding juvenile life. In the first stage, the animal goes through an initial, low-growth phase and, in the second, it develops more rapidly (Figure 1). Individ- 
uals can switch to their second stage at any time step during juvenile life; however, small juveniles grow very slowly in stage 2 due to differences in foraging efficiency and diet breadth (e.g. puncturing vs. macerating food items). Upon reaching a fixed size threshold in stage 2, individuals eventually produce a resistant morph which is analogous to the pupal stage exhibited by many insects. This resistant morph can withstand the harsh conditions at the end of season. Due to the stochastic variation in environmental conditions, however, death can occur anytime prior to the pupa stage due to freezing, desiccation, etc. Death can also occur due to interactions with predators (hereafter, predators also includes parasites). We refer to the total amount of predator-generated mortality as $k$. $k$ may vary across stages when the predators employ different search strategies to attack the prey in one of the two juvenile stages (e.g. different search rules, different search images, etc.). In general, however, predators in our scenario prefer to exploit prey in their second stage (i.e. $k_{2} \geq k_{1}$ ). Thus, growth rates may be higher but they must be traded off against higher stage 2 predation rates as discussed in the Introduction. The basic question then is: Given the aforementioned trade-off, at which point in its juvenile life should an individual of a given size switch from stage 1 to stage 2 ? This question is complicated by several factors that are common to many biological systems. First, prey are not uniform. Their initial size state varies independent of genotype, and may be critical to the growth and survival of the individual, since, as noted above, growth rate is size-dependent (i.e. small individuals perform poorly in stage 2) and predation risk may depend upon relative density and thus the number of individuals who have switched to stage 2 (i.e. predation can be frequency-dependent). Within a single genotype, initial size is assumed to be normally distributed due to a maternal effect (we assume no heritability); this effect is the sole source of variation in initial size among individuals. Because time steps are discrete (16 in our scenario) and growth is continuous, individuals enter into the resistant morph stage at varying sizes; larger individuals may be more fecund and/or vigorous as adults. Second, as noted above, predation can take many forms and thus may favor different strategies regarding switching between stages.

\section{The model}

We consider four scenarios to analyze the expected outcome with and without predation, and with and without frequency dependence. In the first scenario, there is no predation (NP); this scenario serves to provide baseline data on optimal switch-time due to growth constraints. In the second, there is no frequency dependence in predation risk (which, as noted above, could be either predation or parasitism). Thus, within each stage, there is constant predation (CP). In the third scenario (victim or $\mathrm{V}$ ), predation risk during stage 2 depends on the number of prey or victims at this stage (Figure 2). In the fourth scenario (victim-predator or VP), predation risk depends not only on the density of the prey, but also on the preference of the predators for stages 1 and 2 . Thus, the predators in this scenario evolve certain rules to adjust their distribution to the relative density of prey in the two stages. Therefore, the first two scenarios represent a situation without any game, the third involves a game among prey, and the fourth includes a game between prey and predators as well as a game among prey. These scenarios can be applied to various biological systems, for example the developmental stages of leafmining moths (McGregor, 1996), where the time horizon is winter freezing, or developmental stages of anurans, where the time horizon is the desiccation of a temporal pond (Richmond, 1947). In both systems, predation may be highly habitat- or developmental-stage-dependent: moth larvae are preferentially parasitized by a wasp in a particular stage (McGregor, 1996) and different stages of anurans are differentially preyed upon by snakes (Wassersug and Sperry, 1977; Arnold and Wassersug, 1978).

To include both frequency and state dependence in our model, we employed a genetic algorithm (Holland, 1975) in which predation can be frequency-dependent (V and VP) and in which state depen- 


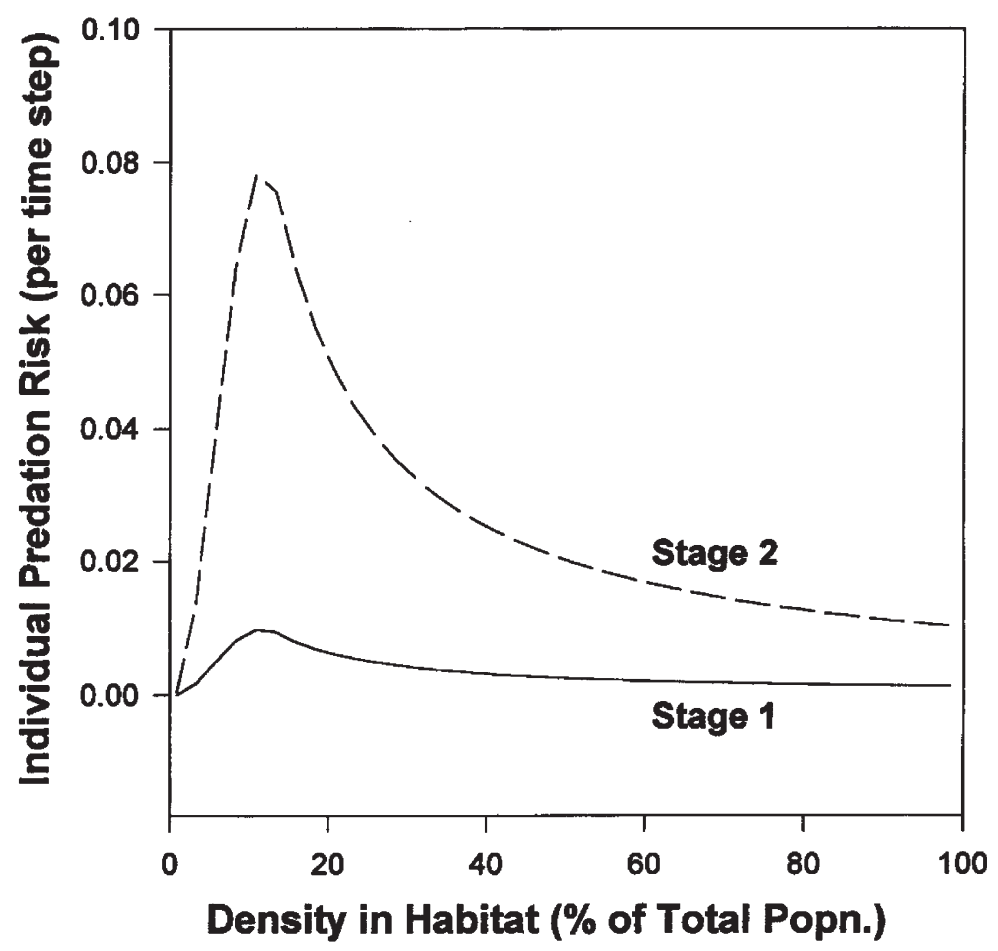

Figure 2. Predation risk per time step as a function of density (\% of total population of prey animals in the stage) for the victim model.

dence is explicitly represented by size at birth. Genetic algorithms reach optimal solutions by searching the enormous space of potential solutions mimicking the process of evolution in biological systems (Davis, 1987, 1991; Goldberg, 1989). Despite their name, genetic algorithms do not attempt to model the genetic evolution of characters; rather, they were developed to find optimal solutions to complex problems and have been used in engineering, image processing and machine learning (Goldberg, 1989; Beasley et al., 1993a). Recently, genetic algorithms were used to elucidate complex optimization problems in biology (Sumida et al., 1990). A genetic algorithm typically describes a population of individuals with heritable characteristics. (We refer to these characteristics as the individual's "genotype," but it should be reiterated that the genetic algorithm does not attempt to model genetic evolution.) An initial population (created by random selection of the characteristics of each individual) goes through stages in a manner analogous to natural selection, fitness evaluation, reproduction, mutations and recombinations, which then determine frequencies of genotypes in the next generation.

These stages proceed as follows: (1) A population of individuals of various genotypes is created. (2) Each prey genotype harbors a switching time for each of five birth size classes. Assuming that size at birth is determined by some environmental factor (e.g. the nutritional condition of the mother), these genotypes represent norms of reaction. (3) Each individual lives her life in a simulation wherein survivorship from predation and catastrophe are stochastically determined. (4) The fitness of each individual is assessed based on survival and size at end of season (see Appendix for details). (5) The fitness of each individual is used to determine the probability of inclusion of its genotype in the next generation. (6) A new population is created based on these probabilities, with the additional possibility of combining parts of different genotypes, and of the occurrence of rare random changes at any 
point in the genotype. Across generations, the algorithm moves repeatedly through these stages to "evolve" subsequent populations that are primarily derived from the most successful individuals of the previous generations (Goldberg, 1989; Davis, 1991). The key point in our model that converts the genetic algorithm into a tool to study state dependence lies in the choice of the characteristics under selection; they address the dependence of the time at which switching between stage 1 and 2 occurs as a function of the initial size of the individual. (In the case of the predators in scenario VP, the algorithm addresses preference for stages 1 and 2.) Thus the "genotype" is a series of switching times or norms of reaction - for each initial size, the genotype determines a switching time. The models act in a manner analogous to natural selection to seek the optimal reaction norms, so that individuals carrying one of these reaction norms will do best when summed across all possible initial sizes (weighted by their frequency of occurrence; Stearns, 1992) when a given set of parameter values is employed.

Optimal norms of reaction for the no-predation (NP) model were determined simply by following a growth trajectory starting at the animal's initial size and at each time step comparing the growth in each stage. Since in the absence of predation the optimal switch time is simply the one which maximizes growth, animals will switch stages when growth in stage 2 exceeds growth in stage 1 (see Figure 1).

Each of the three models including predation (CP, V and VP) was solved using the genetic algorithm. Each model was run for 10,000 generations unless otherwise noted (defined as one "run"). Since results obtained in frequency-dependent models may be dependent on initial conditions, we carried out 10 runs for each parameter set on the two frequency-dependent models (V and VP), using different randomly chosen starting populations for each run. In each case, we sought evidence for the emergence of dominant reaction norms (genotypes) which we defined as the most frequent genotype produced during a single run. Other genotypes which remained in the population at significant frequencies (defined as $>5 \%$ ) we defined as "sub-dominant."

To elucidate the importance of state and frequency dependence, we compared the results from each of the four models using a standard set of parameter values and then varied the following parameters in a systematic manner to assess sensitivity of the models to those parameters within each scenario: predation risk, growth rate, initial-size distribution, end-of-season mortality, pay-off from pupal size, and the shape of the predation curve in the $\mathrm{V}$ model.

Although our model generated individual genotypes (usually a single one which spread to encompass the entire population), we noted that such individual reaction norms would be difficult or impossible to measure in biological systems, especially given the individual variation which we assume. Therefore, we present our main results in terms of two population level statistics: the time at which $50 \%$ of the population has switched stages (defined as the $S_{50}$ ), and the number of time steps required for the majority of the population to switch. When a single genotype making up $>95 \%$ of the population produces behavior in which $>95 \%$ of the population switches in a single time step, we define this as a "dilution strategy." In an effort to assess the stability of the dominant genotypes generated, we also analyzed the results in terms of the number of different dominant genotypes seen across 10 runs, and the presence or non-presence of "sub-dominant" genotypes.

\section{Results}

\section{No predation model}

Results of the no-predation (NP) model with the basic parameter set are shown in Figure 3. The population switches stages over a total of three time steps, with the $S_{50}$ occurring between time steps 6 and 7. As noted above, this simple model represents the maximum growth rate which can 


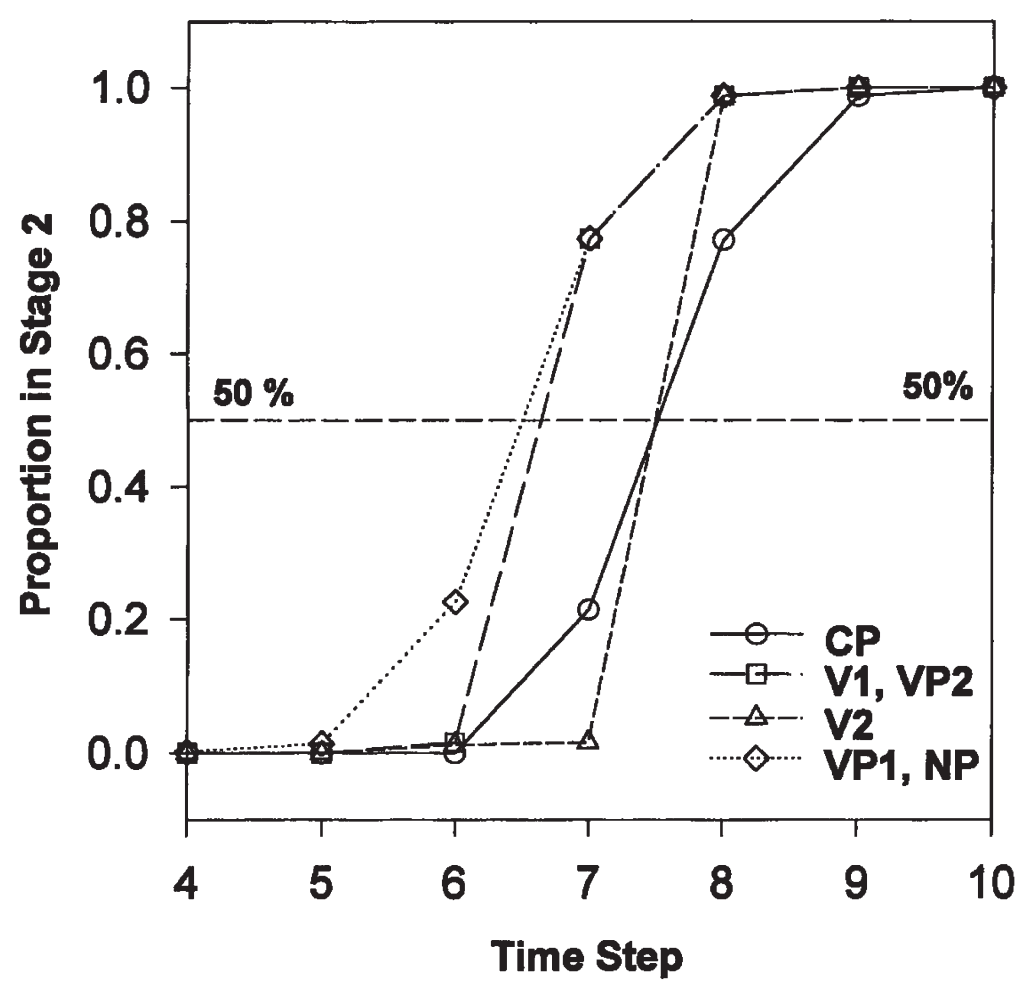

Figure 3. Results of the four scenarios using the basic parameter set. Results are represented in terms of the proportion of the population in stage 2 at each time step. Constant predation (CP) and no predation (NP) scenarios produce only one reaction norm, while the victim model produces two (V1, V2), as does the victim- predator model (VP1, VP2). We define the time at which $50 \%$ of the population has switched stages as the $S_{50}$.

be achieved under a given set of conditions when there is no trade-off with predation. It is of interest as a "benchmark" to which other model results can be compared, illustrating the effect of predation risk.

\section{Constant predation model}

A single dominant reaction norm is generated by the constant predation model (Figure 3, CP). Compared to a population adopting the NP strategy, the $S_{50}$ is shifted approximately one time unit later. Individuals in all but the largest size class switch one time step later (Table 1). This results in slightly smaller sizes in the resistant morph (3.151 vs. 3.209, weighted average across all initial-size classes). The later switch times do not result in longer times to reach the resistant morph, however, and so spending less time in the second stage, with its higher predation risk, increases survival in the CP model (Table 1). The optimal strategy in this model trades off some growth potential to reduce predation risk. When total predation risk is manipulated (maintaining the same relative risk in the two stages), we see a positive correlation between predation risk and $S_{50}$ (Figure 4).

A dilution strategy would offer no benefit in the CP model, since predation risk is constant at all prey densities, and no tendency to switch in a single time step is observed. 


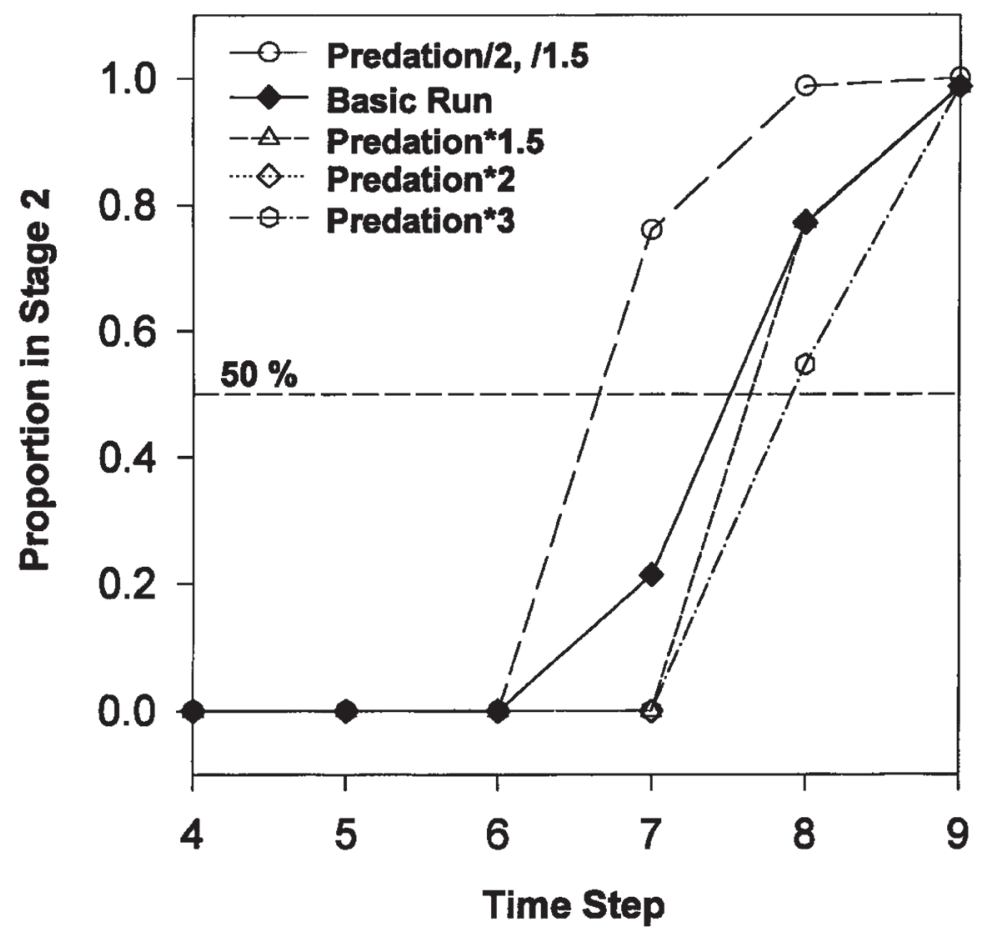

Figure 4. Effect of varying predation risk in the constant predation model. Predation risk was varied by a constant factor in the two stages. This caused the prey animals to switch stages later.

Table 1. Time steps at which the individuals of different norms of reaction (size class 1-5) in the models without predation (NP), with constant predation (CP), the victim game (VI, V2) and the victim-predator game (VP1, VP2) switch from habitat 1 to habitat 2 (switching time), and at which they enter the resistant morph (pupation time)

\begin{tabular}{|c|c|c|c|c|c|c|c|c|c|c|}
\hline \multirow[b]{2}{*}{ Model } & \multicolumn{4}{|c|}{ Switching time } & \multirow[b]{2}{*}{5} & \multicolumn{5}{|c|}{ Pupation time } \\
\hline & 1 & 2 & 3 & 4 & & 1 & 2 & 3 & 4 & 5 \\
\hline $\mathrm{CP}$ & 10 & 9 & 8 & 7 & 8 & 15 & 14 & 13 & 13 & 13 \\
\hline V1 & 9 & 8 & 8 & 8 & 6 & 15 & 14 & 13 & 13 & 12 \\
\hline $\mathrm{V} 2$ & 9 & 8 & 7 & 7 & 6 & 15 & 14 & 13 & 13 & 12 \\
\hline VP1 & 9 & 8 & 7 & 6 & 6 & 15 & 14 & 13 & 13 & 12 \\
\hline VP2 & 9 & 8 & 7 & 7 & 5 & 15 & 14 & 13 & 13 & 12 \\
\hline VP3 & 9 & 8 & 7 & 6 & 5 & 15 & 14 & 13 & 13 & 12 \\
\hline $\mathrm{NP}$ & 9 & 8 & 7 & 6 & 6 & 15 & 14 & 13 & 13 & 12 \\
\hline
\end{tabular}

\section{Victim model}

Two dominant reaction norms were observed in 10 runs with the victim model (V1, V2, Figure 3), demonstrating sensitivity to initial conditions (starting population). Single runs using the basic parameter set led invariably to a single dominant genotype which comprised $>95 \%$ of the population. (See below for further discussion of stability and co-existence of genotypes.) The V1 genotype is a dilution strategy. Its $S_{50}$ is virtually identical to that seen with the optimal CP genotype, but individual 


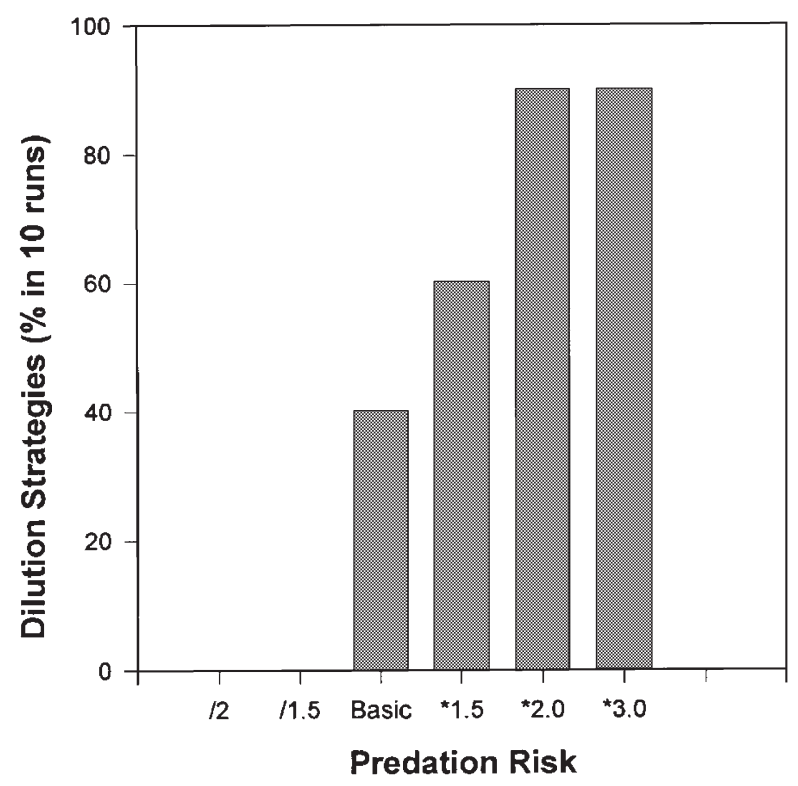

Figure 5. Effect of predation risk on the frequency of dilution strategies in the victim model. Results with this model depend on initial conditions (initial composition of the population), but as predation risk increases by a constant factor in the two stages, dilution strategies (see text for definition) become increasingly common. Results are for 10 runs of the model with different, random, starting populations.

predation risk in each time step is reduced by increasing prey density within a single stage (see Figure 2). The V2 reaction norm is not a dilution strategy by our definition, but the population switches stages over a narrower range of time steps than in NP or CP (two time steps vs. three for $>95 \%$ of the population to switch). The $S_{50}$ is intermediate between NP and CP.

Predation risk influences the likelihood of the population reaching each of these two dominant genotypes (Figure 5). At high predation risk, the dilution strategy V1 becomes dominant in 9 of 10 runs. At low predation risk, the non-dilution strategy V2 becomes dominant in 10 of 10 runs.

\section{Victim-predator model}

The victim-predator model also shows dependence on initial conditions. It generates three dominant reaction norms in 10 runs, but two (VP1, VP3) are identical except for a one-time-step difference in the size class representing the largest individuals (Table 1). Since this size class represents only $1.2 \%$ of the population, we consider these reaction norms to be of the same class, and present results for VP1 and VP2 only. These dominant genotypes are much less stable than those seen in the victim model. The population cycles irregularly between VP1 and VP2, even when run times are doubled to 20,000 generations. Intermediate mixed populations are seen, but are short-lived ( $<50$ generations).

VP1 is identical to NP (Figure 3). Individuals switch at the time which maximizes their growth rate. VP2 is identical to V2 (Figure 3). The population does not adopt a dilution strategy but the range of switch times is compressed from three to two (for $>95 \%$ of the population), and the $S_{50}$ is slightly later compared to NP. In contrast to the victim model, the victim-predator model is almost entirely insensitive to total predation risk. No change in the dominant reaction norms seen after 10,000 generations, or in frequencies of these reaction norms across 10 runs, is observed across the range of predation risk tested (Table 2). 
Table 2. Sensitivity analysis for several model parameters ${ }^{a}$

\begin{tabular}{|c|c|c|c|c|c|c|}
\hline & \multicolumn{3}{|c|}{ Victim model } & \multicolumn{3}{|c|}{ Victim-predator model } \\
\hline & Dom. (\#) & Mult. (Y/N) & Dil. (\%) & Dom. (\#) & Mult. $(\mathrm{Y} / \mathrm{N})$ & Dil. (\%) \\
\hline Basic & 2 & $\mathrm{~N}$ & 40 & 3 & $\mathrm{~N}$ & 0 \\
\hline Pred./2.0 & 1 & $\mathrm{~N}$ & 0 & 3 & $\mathrm{~N}$ & 0 \\
\hline Pred./1.5 & 2 & $\mathrm{~N}$ & 0 & 3 & $\mathrm{~N}$ & 0 \\
\hline Pred.*1.5 & 2 & $\mathrm{Y}$ & 60 & 3 & $\mathrm{~N}$ & 0 \\
\hline Pred.*2.0 & 2 & Y & 90 & 3 & $\mathrm{~N}$ & 0 \\
\hline Pred.*3.0 & 3 & Y & 90 & 3 & $\mathrm{~N}$ & 0 \\
\hline GR/1.1 & 1 & $\mathrm{~N}$ & 0 & 5 & $\mathrm{~N}$ & 0 \\
\hline GR*1.5 & 10 & $\mathrm{Y}$ & 70 & 8 & Y & 40 \\
\hline $\mathrm{GR} * 2.0$ & 10 & $\mathrm{Y}$ & 60 & 9 & $\mathrm{Y}$ & 40 \\
\hline Init. size /1.1 & 2 & $\mathrm{Y}$ & 100 & 7 & $\mathrm{Y}$ & 0 \\
\hline Init. size *1.5 & 5 & Y & 40 & 2 & Y & 0 \\
\hline Init. size *2.0 & 7 & $\mathrm{Y}$ & 10 & 9 & $\mathrm{~N}$ & 20 \\
\hline EOS risk*0.8 & 2 & Y & 50 & 3 & $\mathrm{~N}$ & 0 \\
\hline EOS risk*0.6 & 4 & $\mathrm{Y}$ & 10 & 3 & $\mathrm{~N}$ & 0 \\
\hline EOS risk*0.4 & 3 & $\mathrm{Y}$ & 0 & 4 & $\mathrm{~N}$ & 0 \\
\hline Flat pred. ${ }^{\mathrm{b}}$ & 2 & $\mathrm{Y}$ & 0 & 2 & $\mathrm{~N}$ & 0 \\
\hline $\mathrm{FA}=0.0^{\mathrm{c}}$ & 1 & $\mathrm{~N}$ & 0 & 9 & $\mathrm{Y}$ & 0 \\
\hline $\mathrm{FA}^{*} 0.5$ & 2 & $\mathrm{~N}$ & 90 & 5 & $\mathrm{Y}$ & 0 \\
\hline $\mathrm{FA}^{*} 0.75$ & 2 & Y & 70 & 3 & $\mathrm{~N}$ & 0 \\
\hline $\mathrm{FA}^{*} 1.2$ & 3 & $\mathrm{~N}$ & 10 & 3 & $\mathrm{~N}$ & 0 \\
\hline $\mathrm{FA}^{*} 1.5$ & 3 & $\mathrm{~N}$ & 0 & 3 & $\mathrm{~N}$ & 0 \\
\hline
\end{tabular}

a. Dom. represents the number of dominant solutions seen over 10 runs. Dominant solutions are defined as the most frequent genotype produced in a single run of the model (10,000 generations). Mult. (yes/no) indicates whether any single run of the model produced at least two genotypes with frequency $>5 \%$. Dil. indicates the percentage of the runs which produced dominant genotypes which represent dilution strategies ( $>95 \%$ switching in a single time step). Predation risk (Pred.) and growth rate (GR) are varied by altering the (different) values in both habitats by the same factor.

b. Flat pred. represents a change in the predation curve for the victim model to one with constant high predation at low prey density (no change at high density).

c. FA (fitness advantage) is a parameter controlling the effect of size at pupation on fitness. FA $=0$ represents no effect of size on fitness, and FA*1.5 represents greatest effect of size on fitness (see Appendix).

\section{Additional sensitivity analysis}

\section{Constant predation model}

Increasing initial sizes across the range basic/1.1 to basic*2.0 leads to earlier $S_{50}$ values, which is consistent with the negative correlation between switch time and initial size seen across all models using the basic parameter set. However, increasing growth rates across the same range leads to later $S_{50}$ values. The model is relatively insensitive to reductions in end-of-season risk, but when end-of-season mortality is reduced substantially (basic ${ }^{*} 0.4$ ), making growth less important relative to predation, $S_{50}$ is later. Changing the pay-off from size at end of season (as a proportion of fitness) has only a small effect on $S_{50}$ in the CP model. 
Changing parameter values has no effect on the stability of this model (as measured by number of dominant reaction norms seen across 10 runs, and maintenance of multiple reaction norms within a single run). Each parameter set produces a single dominant reaction norm with no dependence on initial conditions. Dilution strategies are not observed under any conditions tested.

\section{Victim model}

Growth rates, initial size, end-of-season mortality and the shape of the predation curve had no consistent effect on $S_{50}$. When the size pay-off is reduced, $S_{50}$ is later in the victim model.

Growth rate and initial size have opposite effects on the proportion of dilution strategies: as growth rate increases, the proportion of dilution strategies also increases; however, as initial size increases, the proportion of dilution strategies declines, as it does if end-of-season risk is reduced. Changing the shape of the predation curve (constant and high at low prey densities instead of increasing over this density range) led to the elimination of dilution strategies.

Increasing growth rate and initial size both led to an increased number of dominant strategies across runs, and increased occurrence of multiple reaction norms within a run. For details of the sensitivity analysis, see Table 2 .

\section{Victim-predator model}

As with the victim model, no consistent effects on $S_{50}$ can be seen by manipulating growth and initial size. $S_{50}$ in this model is insensitive to reductions in end-of-season mortality. $S_{50}$ is also insensitive to size pay-off; only when size pay-off is entirely eliminated is $S_{50}$ delayed.

Increasing growth rates leads to more dominant reaction norms across runs and multiple genotypes within runs. The effect of initial size on these measures of stability is inconsistent. Dilution strategies are generated both by high growth rates and large initial sizes.

\section{Co-existence of multiple reaction norms}

In our model runs, stable co-existence of multiple reaction norms was never observed. In the victim and victim-predator models, however, two dominant reaction norms are produced by different starting conditions. To test whether two norms of reaction can co-exist, we ran the simulation portion of our model with different starting frequencies of V1:V2 for the victim model and VP1:VP2 for the victim-predator model. We observed co-existence for neither model, with one reaction norm typically becoming dominant ( $>95 \%$ of population) within a few dozen generations. In the victim-predator model, VP2 always outcompetes VP1; in the victim model, the outcome depends on initial frequencies. V1 goes extinct if $\mathrm{V} 2$ is $\geq 41 \%$.

\section{Discussion}

In our model, we assumed that there is a fitness advantage to pupating at a larger size; this makes biological sense because larger individuals have higher fecundity, mating success or dispersal ability (Roff, 1991). Therefore, in the absence of predators, evolution should favor individuals that maximize their growth, similar to our NP model results. In addition, the sooner they reach their final size, the more likely they are to pupate before the end of season (EOS risk). Individuals following the optimal growth trajectory switch to habitat 2 as soon as they reach the size at which the growth rate in 
habitat 2 exceeds that in habitat 1 . When there are different predation risks in habitat 1 and 2, however, there is a trade-off between the mortality risk, EOS risk and a high pupal size. The risk of death increases exponentially as the end of the season approaches. This results in the increase in end-of-season mortality with later pupation times. Therefore, genotypes that pupate as soon as possible should realize high fitness. As growth rate is dependent on current size, individuals that are born small have a disadvantage throughout their whole life. For example, the smallest individuals cannot pupate before time step 15 and suffer from a 50\% probability of death due to end-of-season effects. In contrast, in all of our models, the mortality due to predation for the whole larval development is $<35 \%$. In all the models, the dominant genotypes have almost identical pupation times, indicating that EOS determines the time at pupation and the trade-off between high pupal size and predation risk determines the switch time (several switch times often produce the same pupation time). Without an advantage to pupating at a large size, it would be favorable, of course, to switch as late as possible to minimize the predation risk (see sensitivity analysis).

We can use the comparison between the no predation model and the other models to estimate the influence of predation on the norms of reaction. With the exception of VP1, all norms of reaction lead to later switch times than the maximum growth would suggest. Because predation risk in habitat 2 is higher than in habitat 1, it might be better to diverge from maximum growth and switch late to habitat 2 to reduce predation. This means that, on the one hand, individuals following these norms of reaction decrease their pupal size, but on the other, they spend less time in the habitat with high predation risk resulting in a reduction in the risk of being preyed upon.

The difference between the results of the CP and the NP models is equivalent to the predictions from the models of Werner and Gilliam (1984) and Werner (1986). These authors state that, in a system of two ontogenetic habitats, the inclusion of predation risk (higher in the second habitat) will delay the time of switching. Another comparison that can be made to these studies relates to growth rates: increasing growth rate in our constant predation model delayed switching time, as predicted by Werner and Gilliam (1984) and Werner (1986).

Our models incorporate some of the factors that Werner (1986) suggests adding to his model, such as the catastrophic mortality at the end of the season and the density dependence in the mortality rate. This last addition leads to our prediction of synchronization in the switching time in the victim model. Other additions in our models include the version with a game between prey and predator, and the effect of the initial size, which adds state dependence to the models.

Predation risk is an important component in the constant predation and the victim game models. When predation risk is not dependent on prey frequency, prey can only reduce their predation risk by switching later (see Table 1). However, if predation risk is frequency-dependent, then dominant genotypes can also reduce predation risk by dilution, if most of the population switches at the same time. This is equivalent to the dilution effects in groups (Turner and Pitcher, 1986; Wrona and Dixon, 1991), which has been documented in the metamorphosis of anurans (Arnold and Wassersug, 1978). With rising predation risk in our model, this strategy becomes more frequent (Figure 5). Nevertheless, when the predators are also allowed to adjust their strategy according to the prey's distribution, the advantage of dilution disappears. This result is equivalent to predictions of other models that incorporate predator attraction to large groups of prey (Turner and Pitcher, 1986), but is often ignored when the dilution effect is considered (e.g. Treherne and Foster, 1982).

Of the two dominant strategies produced by the victim-predator game, VP1 is closest to the optimal growth (NP), but VP2 is not much different. This suggests a low influence of predation risk on the norm of reaction of the prey when such games operate. The influence of the predators on the switching behavior of the prey remains low, even when the overall predation risk is increased (see sensitivity analysis). This low influence does not mean that predation has no impact (VP1 and VP2 have low 
survival compared with the survival in the constant predation model and prey game). In the victimpredator game model, the predators adapt their preference for exploiting individuals in habitat 1 or 2 according to the timing of habitat switch by the prey. Because the preference of the predators is dependent on the switching times of prey, each strategy of the prey to minimize predation risk will be counteracted by the changing preference of the predators. Therefore, genotypes that simply maximize their growth outperform predation-averse strategies.

Obviously, the inclusion of density dependence, whether in the victim game or the victim-predator game, resulted in unique predictions. Similarly, the influence of state dependence was obvious in all of the model versions, because the strategies for each size class were markedly different, and there were interesting interactions with the effects of the various game situations. We can thus conclude that both state and frequency dependence provide useful insights in understanding ontogenetic theory. While there is ample evidence for the importance of state dependence in natural systems (e.g. Godin and Sproul, 1988; Mangel and Roitberg, 1989), it is not yet clear in which systems game situations are operating. To decide whether to apply frequency dependence considerations for a specific system, we need to determine if animals in that system play any of the games. We could use our most general results which distinguish between game and non-game situations. In theory, this can be done by varying the overall predation risk and analyzing the patterns in the resulting switching time distributions. Distinguishing in nature between the different kinds of distribution may, however, be difficult, because the difference in the switching pattern of the various models is only visible within two time steps or $12.5 \%$ of the whole season (Figure 3). Therefore, longer intervals between density samples in both habitats might lead to incorrect conclusions. One might be able to identify the kind of predatorprey interactions by manipulating the overall predation risk, and determining the intensity of directional selection on the switching strategies by measuring the selection differential. In our models, selection operates primarily by means of differential survival, and therefore the selection differential in natural systems can be calculated as the difference between the mean value of the switching strategy in the surviving individuals and the mean value of the switching strategies in all individuals before selection occurs (Rausher, 1992). There are two components of the strategies that can be quantified: the mean and the variance of the switching time distribution. Selection for decreased variance is selection for dilution, based on our earlier terminology. With these quantities in mind, we can use the following decision tree to select the most suitable model:

(a) Is there selection for decreasing the variance of the switching time distribution when the predation risk is increased?

- yes: victim model

- no: go to $b$

(b) Is there selection for increasing the mean switching time when the predation risk is increased?

- yes: model with constant predation risk

- no: victim-prey game model

Acknowledgments - We thank D. Bartlett, P. Harrald, M. Johnston, R. McGregor, I. Robertson, M. Spencer, D. Tyre and E. van Randen for helpful discussions. G. Smith provided useful references. This work was supported by an NSERC operating grant to B.D.R. 


\section{References}

Abrams, P. A. and Rowe, L. (1996) The effects of predation on the age and size of maturity of prey. Evolution 50, 1052-1061.

Antonisse, J. (1989) A new interpretation of schema notation that overturns the binary encoding constraint. In Proceedings of the 3rd International Conference on Genetic Algorithms (J. D. Schaffer, ed.), pp. 86-91. MorganKaufmann, San Mateo, CA.

Arnold, S. J. and Wassersug, R. J. (1978) Differential predation on metamorphic anurans by garter snakes (Thamnophis): Social behaviour as a possible defence. Ecology 59, 1014-1022.

Beasley, D., Bull, D. R. and Martin, R. R. (1993a) An overview of genetic algorithms. Part 1: Fundamentals. Univ. Comp. 15, 58-69.

Beasley, D., Bull, D. R. and Martin, R. R. (1993b) An overview of genetic algorithms. Part 2: Research topics. Univ. Comp. 15, 170-181.

Bouskila, A. (1995) Interactions between predation risk and competition: A field study of kangaroo-rats and snakes. Ecology 76, 165-178.

Davis, L. (1987) Genetic Algorithms and Simulated Annealing. Pitman, London.

Davis, L. (1991) Hybridization and numerical representation. In Handbook of Genetic Algorithms (L. Davis, ed.), pp. 61-71. Van Nostrand Reinhold, New York.

Godin, J.-G. J. and Sproul, C. D. (1988) Risk taking in parasitized sticklebacks under threat of predation: Effects of energetic need and food availability. Can. J. Zool. 66, 2360-2367.

Goldberg, D. E. (1989) Genetic Algorithms in Search, Optimization and Machine Learning. Addison-Wesley, Reading, MA.

Holland, J. H. (1975) Adaptation in Natural and Artificial Systems: An Introductory Analysis with Applications to Biology, Control, and Artificial Intelligence. University of Michigan Press, Ann Arbor, MI.

Iwasa, Y. and Levin, S. A. (1995) The timing of life history events. J. Theor. Biol. 172, 33-42.

Ludwig, D. and Rowe, L. (1990) Life history strategies for energy gain and predator avoidance under time constraints. Am. Nat. 135, 686-707.

Mangel, M. and Roitberg, B. D. (1989) Dynamic information and oviposition decisions in a fruit fly. Ecol. Ent. 14, 181-189.

McGregor, R. (1996) Seasonal changes in selection pressures in a leafminer. Evolution 50, 1579-1584.

Murdoch, W. M. (1994) Population regulation in theory and practice. Ecology 75, 271-287.

Rausher, M. D. (1992) Natural selection and evolution of plant-insect interactions. In Insect Chemical Ecology: An Evolutionary Approach (B. Roitberg and M. Isman, eds.), pp. 20-88. Chapman \& Hall, New York.

Richmond, N. D. (1947) Life history of Scaphiophus holbrookii (Harlan). Part 1: Larval development and behavior. Ecology 28, 53-67.

Roff, D. (1991) Life history consequences of bioenergetic and biomechanical constraints on migration. Am. Zool. 31, 205-215.

Rowe, L. and Ludwig, D. (1991) Size and timing of metamorphosis in complex life cycles: Time constraints and variation. Ecology 72, 413-427.

Smith-Gill, S. J. and Berven, K. A. (1979) Predicting amphibian metamorphosis. Am. Nat. 113, 563-585.

Stearns, S. C. (1992) The Evolution of Life Histories. Oxford University Press, New York.

Sumida, B. H., Houston, A. I., McNamara, J. M. and Hamilton, W. D. (1990) Genetic algorithms and evolution. J. Theor. Biol. 147, 59-84.

Treherne, J. E., and Foster, W. A. (1982) Group size and anti-predator strategies in a marine insect. Anim. Behav. $32,536-542$. 
Turner, G.F. and Pitcher, T. J. (1986) Attack abatement: A model for group protection by combined avoidance and dilution. Am. Nat. 128, 228-240.

Wassersug, R. J. and Sperry, D. G. (1977) The relationship of locomotion to differential predation on Pseudacris triseriata (Anura: Hylidae). Ecology 58, 830-839.

Werner, E. E. (1986) Amphibian metamorphosis: Growth rate, predation risk, and the optimal size at transformation. Am. Nat. 128, 319-341.

Werner, E. E. and Gilliam, J. F. (1984) The ontogenetic niche and species interactions in size-structured populations. Annu. Rev. Ecol. Syst. 15, 393-425.

Werner, E. E., Gilliam, J. F., Hall, D. J. and Mittelbach, G. G. (1983) An experimental test of the effects of predation risk on habitat use in fish. Ecology 64, 1540-1548.

Wilbur, H. M. and Collins, J. P. (1973) Ecological aspects of amphibian metamorphosis. Science 182, 1305- 1314.

Wrona, F. J. and Dixon, R.W.J. (1991) Group size and predation risk: A field analysis of encounter and dilution effects. Am. Nat. 137, 186-201.

\section{Appendix: Additional assumptions and details of the model}

\section{End of season}

The end of season is a stochastic catastrophe in which all individuals die except those which are already in the resistant phase (e.g. a pupa of the leafminer moth, or a terrestrial anuran, which are not affected by freezing or by desiccation of the pond, respectively). We assumed that there is a probability $\operatorname{pr}\{\mathrm{EOS}\}$ associated with each time step for the occurrence of this catastrophe; the probability increases and reaches 1 at the last time step according to the function:

$$
\operatorname{pr}\{\mathrm{EOS}\}=\exp (t) / \exp \left(t_{\max }\right)
$$

where $t$ is the current time step $\left(t=1, t_{\max }\right)$.

\section{Initiation of a generation}

At the beginning of each generation, we always start with 120 genotypes. The characteristics of each genotype are randomly chosen at the first generation; in subsequent generations, the characteristics are determined by the genetic algorithm (the best genotypes are passed on to the next generation). However, initial size of the juveniles in our model is not an inherited character. The distribution of the initial size of individuals at the establishment of each generation is close to normal with five size classes, where the smallest and largest size classes are at 3 standard deviations from the mean (in the basic run the mean was set to 0.16 , S.D. $=0.02$; for comparison, the critical size above which the juveniles enter the resistant stage was 3 ).

\section{The genetic representation}

The characteristics of each individual are contained in a string of five integers, the "chromosome" in genetic algorithm terminology (Goldberg, 1989). Early genetic algorithm models used only binary coding for the characters (i.e. only the digits 0 or 1 would appear in the string representing the chromosome) (Holland, 1975; Goldberg, 1989). Recently, however, it has been argued that non-binary coding may have advantages (Antonisse, 1989; Davis 1991). In our model, we coded with integers between 1 and 16 (representing the time step for switching between stage 1 and 2), because we noticed that, when we used binary coding, there were occasions in which the model would converge on local optima, unable to cross to higher optima. 
Each of the five integers in a string representing a chromosome determines the switching time for an individual at one of the five initial sizes. Thus if the string is 98878 , for example, the smallest size class will switch at the 9th time step, the next two size classes, as well as the largest, will switch at the 8th, and the fourth size class will switch at the 7th time step. Recall that each individual may start at any of the initial sizes with a certain probability, thus the fitness of a genotype is a weighted average of five fitnesses, and the weighting is done according to the probabilities associated with each of the initial sizes.

\section{Fitness evaluation}

At the end of each generation, the fitness of each individual is assessed, in order to create the next generation from the genotype of the individuals with the highest fitness. Fitness in our model is based on survival and on size at the end of the season. The probability of survival (which is updated after each time step, for each individual, according to its probability of being preyed upon or killed by the EOS risk) is multiplied by a pay-off from size at pupation, to form the fitness. To create the next generation, pairs of individuals from the old generation are drawn randomly, with a probability proportional to their fitness. The genotypes of two such individuals is then combined (employing crossovers and mutations, as described below) to form the basis for the genotypes of two new individuals that will be part of the next generation.

\section{Crossovers and mutations}

Genetic algorithms employ crossovers (combining parts from two different genotypes) and mutations (a random change in the genotype) as central tools in the search for new genotypes and for optimal solutions (Goldberg, 1989). The rate of mutations is one of the important parameters that determines how well the genetic algorithm performs (Beasley et al., 1993b). In our model, a genotype had a probability of 0.5 for the occurrence of a crossover at a random point along the string of five integers. After the formation of the offspring genotype, a mutation could occur at each of the five integers with a probability of 0.001 . When a mutation occurred, an integer between 1 and 16 was randomly selected as a replacement. In our model, higher levels of mutation disrupted the convergence of the model, while lower values seemed to miss the formation of certain high-fitness genotypes. 